\title{
Research on jitter prevention technology of large mechanical control process
}

\author{
Ren Guoqiang \\ Sichuan Vocational and Technical College,629000
}

Keywords: singular perturbation method; jitter; simulated annealing

\begin{abstract}
. in the research of jitter prevention technology of large mechanical control process, with the current algorithm to prevent jitter, the circuit cannot achieve a desired steady state in an instant, and of poor stability. For this, a jitter prevention method of large mechanical control process based on improved simulated annealing algorithm is proposed, simulated annealing algorithm is utilized to determine the initial state of the jitter, and calculate the increment of evaluation function and corresponding function, fusing with singular perturbation method to decompose the system into two subsystems of slow and fast changing, and singularly perturbed model are formed separately. For the slow changing subsystem, a simulated annealing algorithm of strong robustness is adopted to prevent jitter, while the fast changing subsystem employs linear quadratic optimal controller based on reduced order state observer, effectively prevent jitter in the process of large mechanical control. Simulation results show that jitter prevention method of large mechanical control process based on the improved simulated annealing algorithm have high efficiency, strong robustness.
\end{abstract}

\section{Introduction}

With the continuous improvement of the construction level, large-scale machinery equipment is applied widespread in machinery, chemical industry, metallurgy, building materials and other industrial sectors 1.2. But space environment of large mechanical equipment is particular, when machinery is in control, elastic jitter will happen between two contacts, so as to produce interference pulse to damage the service life of large machinery seriously 3.4.5. Jitter prevention technology is an effective way to solve this problem, and attracts attention from a lot of experts and scholars 6. At present, the commonly used jitter prevention algorithm mainly includes fuzzy algorithm, particle swarm algorithm and genetic algorithm. But by using the algorithm to prevent large machinery jitter, the circuit cannot achieve a desired steady state in an instant, the stability is poor.

Aiming at the above problems, a jitter prevention method of large mechanical control process based on improved simulated annealing algorithm is proposed. Simulation results show that jitter prevention method of large mechanical control process based on the improved simulated annealing algorithm have high efficiency, strong robustness.

\section{Jitter prevention principle of large mechanical control process}

Assuming in working process of large scale mechanical jitter preventing circuit, the initial state of large mechanical control set at contact $m$, the input signal is $\bar{R}=0, \bar{S}=1$, the basic $R S$ flip-flop function shows, at this time the circuit function is set to 0 . When the total output $Q$ is 0 , control is off. The control process is divided into three stages from fault to conduction:

Firstly, when control left the contact $m$ and has not yet reached the contact $n$, the circuit input signals $\bar{R}=1, \bar{S}=1$. The basic $R S$ flip-flop function shows, at this time the circuit functions is maintain, and the total output state does not change,. When the $Q$ terminal is kept at 0 , control remains disconnected.

Secondly, when control reach the contact point $N$, the circuit input signal changes into $\bar{R}=1$, $\bar{S}=0$. The basic $R S$ flip-flop function shows, at this time the function of the circuit is set as 1 , 
the total output $Q$ state is 1, which shows the control is conducting at the time.

Thirdly, when control reached the contact point $n$, small jitter is produced in a short time, which caused connection and off with the $n$ point many times. The input signal of flip-flop switch between $\bar{R}, \bar{S}$, which means between 1, 0 and 1, 1 fast, so as to become the burr phenomena on the waveform. But this change of input signal does not affect the state of output, the basic $R S$ flip-flop function shows, function of large mechanical circuit is switched between arranged 1 and maintain. So the total output $Q=1$ status will not change, that is, the switch is always in the conducting state.

\section{Jitter prevention optimization method principle of large mechanical control process}

\subsection{Determination of the initial state and calculation of its evaluation function}

Firstly, during jitter prevention optimization process, the initial state of jitter is confirmed with the simulated annealing algorithm, and the increment of evaluation function and corresponding function are calculated. The steps are as follows:

The different arrangement modes of elements in generating jitter state is corresponding to different conditions, the following evaluation function is chosen to distinguish different state of the jitter:

$$
E=\sum_{i} \sum_{p, q} \frac{1}{r\left(p_{i}, q_{i}\right)^{2}}
$$

Wherein, $E$ represents the evaluation function, $i$ represents a position value at the specified state, $p_{i}$ represents the position of value less than $i$ at specified state, $q_{i}$ represents the position of value less than $i$ at specified state, $r\left(p_{i}, q_{i}\right)^{2}$ represents the square of Euclidean distance between $p_{i}$ and $q_{i}$. Obviously, with different $E$ values, the corresponding modes of jitter state are different.

The value of a location at $i$ state, can be obtained by calculating evaluated function $E_{0}$ of initial state of jitter:

$$
E_{0}=\sum_{k=1}^{62} \sum_{i=1}^{k} \sum_{j=0}^{i=1} r j, t
$$

The location of value less than $i$ under the condition $p_{i}$, can be calculated according to the evaluation function $E_{0}$ as follows:

$$
\sum_{i=1}^{8^{2}-1} C(i, 2)=\frac{8^{2}\left(8^{2}-1\right)\left(8^{2}-2\right)}{6}=o\left(8^{6}\right)
$$

The evaluation function part of jitter state influenced by point $v_{1}$ can be calculated as follows:

$$
\sum_{i=v_{1}+1}^{N_{2}-1} \sum_{j=0}^{i-1}=r j, v_{1}
$$

Accordingly, the total amount of computation of $\Delta E$ is:

$2\left[\left(v_{1}+(v 1+1)+\ldots+\left(N^{2}-2\right)\right)\right]=\left(v^{2}+\left(v^{2}+1\right)\right)$

If $v=\min \left\{v_{1}, v_{2}\right\}$, then the above formula is not greater than $4[v+(v+1)]$, the worst case is $v=0$, the calculation amount is: 
$4\left(0+1+2+\cdots+\left(N^{2}-2\right)\right)=2\left(N^{2}-2\right) *\left(N^{2}-2\right)$

The evaluation function part of the state influenced by point $v_{1}$ can be calculated as follows: $\left(N^{2}-1-v 1\right) \sum_{i=0}^{V 1-1} r j, v_{1}=\sum_{V 1-1}^{N^{2}-2}\left(N_{\frac{1}{2}}^{2}-1-v 1\right)$

Accordingly, computation quantity of $\Delta E$ (measured by the quantity of computed distance) is: $4\left(N^{2}-2\right)=o\left(N^{2}\right)$

According to Metropolis criterion, the increment of corresponding function is calculated by the formula:

$t_{0}=\frac{\Delta f^{+}}{1 n x^{-1}}$

Among them, $\Delta f^{+}$is average increment of target function, $x$ is the initial acceptance rate of new solution, which should be close to 1 , the empirical value is between $0.85 \sim 0.95$.

3.2 The realization of jitter prevention optimization method of large mechanical control process

The following formula can be utilized to build singular perturbation model of jitter prevention system of large mechanical control process:

$D(\theta, q)=\left[\begin{array}{ll}D_{r r} & D_{r f} \\ D_{f r} & D_{f f}\end{array}\right]$

The inverse matrix is:

$H=\left[\begin{array}{ll}H_{11} & H_{12} \\ H_{21} & H_{22}\end{array}\right]=\left[\begin{array}{ll}D_{r r} & D_{r f} \\ D_{f r} & D_{f f}\end{array}\right]$

The proportional factor of singular perturbation is $\mu=1 / \min \left\{k_{44}, k_{55}\right\}$, and the new state variable $D(\theta, q)=q / \mu$ is introduced, the singular perturbation model of slow changing subsystem is obtained:

$\bar{\theta}=\bar{D}_{r r}^{-1}\left(-\bar{C}_{r}+\tau_{s}\right)$

Based on the Lyapunov stability theorem, the jitter state of slow changing subsystem converges from any initial jitter state in the control process, which can be expressed by the formula:

$t_{s}=t_{r}+\frac{p}{p-q} \max _{i=1,2,3}\left[\lambda_{i} e_{i}\left(t_{r}\right)^{p /(P-q)}\right]$

Based on the above formula, the coefficient $\epsilon_{\text {of }} \operatorname{sigh}$ function control item $\in \operatorname{sgn}(s)$ has great relationship with time to achieve the control process and degree of jitter. In order to effectively reduce the jitter and ensure the system has fast response, the controller composed of 3 single input, single output simulated annealing control system was introduced to the control system to weigh the value of coefficient $\in$.

For the fast changing subsystem, because $\left(A_{f}, B_{f}\right)$ is completely controllable, the optimal control theory can be utilized, the following formula is employed to construct optimal control performance index function:

$J=\frac{1}{2} \int_{0}^{\infty}\left(Z^{T} Q_{Z}+\tau_{f}^{T} R \tau_{f}\right) d t$

Then, optimal control laws of the fast changing subsystem can be designed as: $\tau_{f}^{T}=-k_{2} Z=-R^{-1} B_{f}^{T} P_{Z}$

If the control torque $\tau_{f}^{T}$ need to be calculated, the real-time value of each state variable in fast 
changing subsystem should be informed in advance. Introducing the state observer to estimate the state variables which are not easy to measure in real time. Calculations are performed by the formula:

$\hat{Z}_{2}=W=\hat{Z}_{2}+\Gamma_{V}$

The above formula can be rewritten as:

$\tilde{z}=\left[\begin{array}{c}y \\ w-\mathrm{T} y\end{array}\right]$

The LQR control law of fast changing subsystem based on the state observer: $\tau_{f}=-k_{z} \widehat{z}=-k_{2}\left[\begin{array}{c}y \\ w-\mathrm{T} y\end{array}\right]$

\section{Experiment and simulation}

In order to prove the validity of the improved simulated annealing algorithm, an experiment is needed. On the Matlab/Simulink platform, the optimization simulation platform was built. Respectively with traditional algorithm and improved algorithm to conduct jitter prevention simulation experiment of large scale mechanical control process, the jitter is random in the control process. The effect of jitter prevention is shown in Figure 1

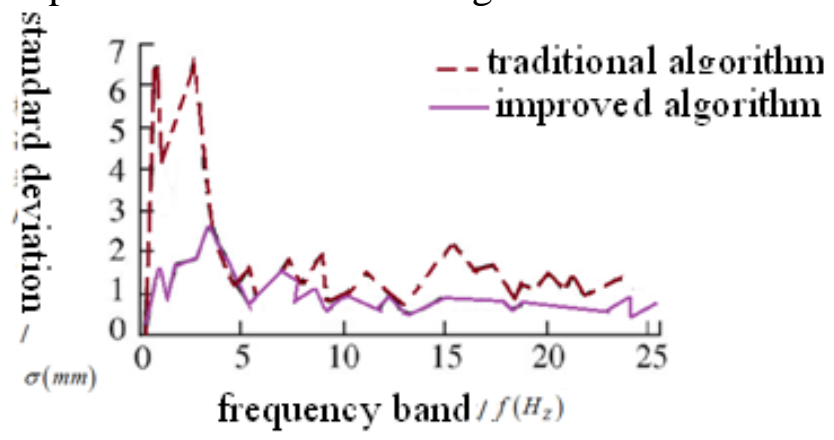

(A) Left sensor

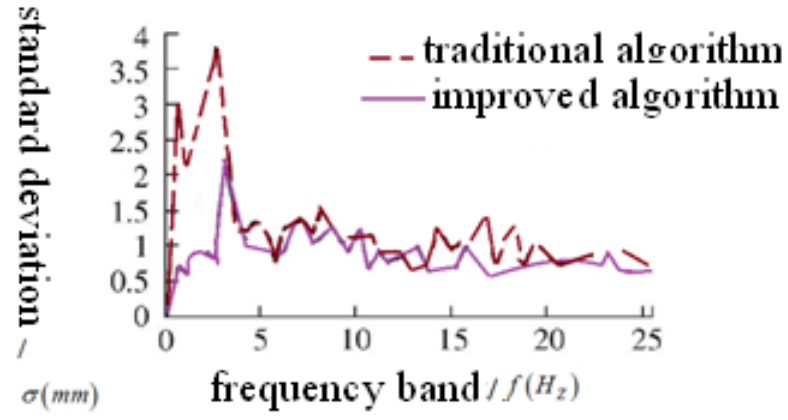

(B) Right sensor

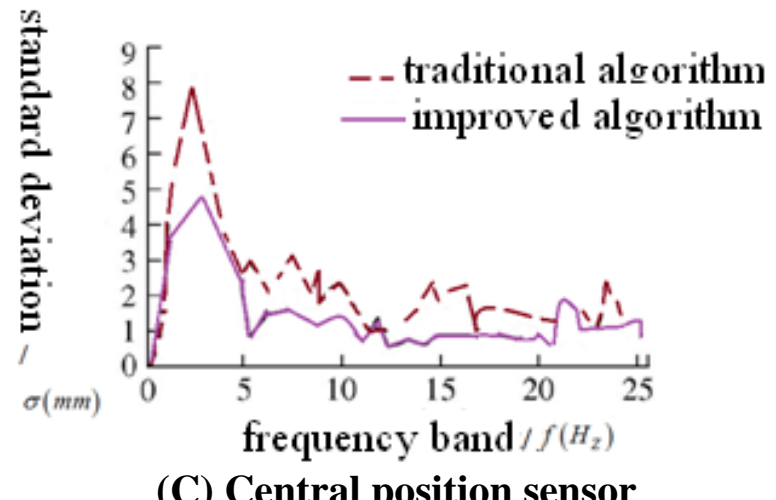

Fig. 1 the control effect of different methods

As you can see from Figure 1, for the slow changing subsystem, a simulated annealing algorithm 
of strong robustness is adopted to prevent jitter, while the fast changing subsystem employs linear quadratic optimal controller based on reduced order state observer, effectively prevent jitter in the process of large mechanical control, with strong robustness.

\section{Conclusions}

In this paper, a jitter prevention method of large mechanical control process based on improved simulated annealing algorithm is proposed, simulated annealing algorithm is utilized to determine the initial state of the jitter, and calculate the increment of evaluation function and corresponding function, fusing with singular perturbation method to decompose the system into two subsystems of slow and fast changing, and singularly perturbed model are formed separately. For the slow changing subsystem, a simulated annealing algorithm of strong robustness is adopted to prevent jitter, while the fast changing subsystem employs linear quadratic optimal controller based on reduced order state observer, effectively prevent jitter in the process of large mechanical control. Simulation results show that jitter prevention method of large mechanical control process based on the improved simulated annealing algorithm have high efficiency, strong robustness.

\section{References}

[1]Zhang Quanfa, Wang Dongya, Fei Guangyan, et al. automatic search method for anti-jitter region of video vehicle monitoring system [J]. Journal of Zhengzhou University: Natural Science Edition.2014.2:59-63.

[2] Zhang Hongyan, Zhao Jun, Zhang Hongjun. Analysis of loop problem caused by the accident general signal [J]. Journal of Hebei Engineering and Technical College.2014.3:36-39.

[3]Liu Haining. Design of digital adjustable voltage comparator based on threshold MCU [J]. Mechanical manufacturing and automation.2013.4:181-183.

[4] Fu Zhen, Li Dong. The application research of analog switch CD4053 in the microcontroller [J]. Technology square.2013.5:137-139.

[5] Zhang Deshu. A simple programmable gain amplifier circuit design [J]. Journal of Chuzhou Vocational and Technical College,.2013.1:62-64.

[6] Wu Wei, Xu Yan, Wang Yulei. The application of IRIG - B code in a combined unit [J]. Power measurement and instrumentation.2014.1:12-16. 